\title{
Ultra-Wideband Schiffman Phase Shifter Designed with Deep Neural Networks
}

This paper was downloaded from TechRxiv (https://www.techrxiv.org).

\section{LICENSE}

CC BY 4.0

SUBMISSION DATE / POSTED DATE

$12-11-2021 / 24-11-2021$

\section{CITATION}

an, sensong; Zheng, Bowen; Tang, Hong; Li, Hang; Zhou, Li; Dong, Yunxia; et al. (2021): Ultra-Wideband Schiffman Phase Shifter Designed with Deep Neural Networks. TechRxiv. Preprint. https://doi.org/10.36227/techrxiv.17003539.v1

$\mathrm{DOI}$

10.36227/techrxiv.17003539.v1 


\title{
Ultra-Wideband Schiffman Phase Shifter Designed with Deep Neural Networks
}

\author{
Sensong An, Bowen Zheng, Hong Tang, Hang Li, Li Zhou, Yunxi Dong, Mohammad Haerinia, \\ Student Member, IEEE and Hualiang Zhang, Senior Member, IEEE
}

\begin{abstract}
This paper presents a novel method for the forward modeling and inverse design of a class of Schiffman phase shifters using Deep Neural Networks (DNNs). Since DNNs are capable of mapping the highly nonlinear correlations between inputs and outputs, we constructed a fully-connected DNN to predict the electromagnetic (EM) responses of Schiffman phase shifters given their physical dimensions. Based on this fast and accurate modeling tool, a cascaded inverse design DNN was then built and trained to achieve instant on-demand phase shifter designs. This approach is versatile and can be easily modified to accomplish different design goals. To demonstrate its efficacy, we trained two DNNs to realize Schiffman phase shifter designs with different bandwidths $(40 \%$ and $60 \%)$ and arbitrary phase shift targets $(0-$ $180^{\circ}$ ). Simulation results and experimental verifications substantiate that their performances are comparable to the stateof-the-art designs. Moreover, we discussed the proposed methods' potential in dealing with design tasks that are non-intuitive and beyond the scope of existing approaches. We envision that this DNN approach can be extended to the design of various EM components including but not limited to antennas, filters, power dividers, and frequency selective surfaces (FSS).
\end{abstract}

Index Terms - Schiffman, wideband phase shifter, deep learning, Deep Neural Networks, inverse design

\section{INTRODUCTION}

$\mathrm{P}$ hase shifters play an important role in beam scanning phased arrays, modulators and wireless communication systems. Ideal phase shifters should provide flat phase shift over wide operating frequency band with low loss. Among various types of differential phase shifters, those realized in planar technology, either stripline [1], microstrip line [2] or substrate integrated waveguide (SIW) [3] are widely adopted due to their low cost, small amplitude imbalance and widebandwidth characteristics. In general, wideband planar phase shifters can be characterized into several categories: Schiffman phase shifters [1, 2, 4-12], loaded-line phase shifters [13-18], broadside coupling phase shifters [19-21] and others [22-24]. Compared with their counterparts, the Schiffman phase shifters are compact in size, easy to fabricate using Print Circuit Board

This paper was submitted for review on Oct. 25, 2021. This work is supported in part by the ONR. (Corresponding author: Hualiang Zhang)

S. An was with the University of Massachusetts Lowell, Lowell, MA 01854 USA. He is now with the Department of Material Science and Engineering, Massachusetts Institute of Technology, Cambridge, 02142 USA (e-mail: ssan@mit.edu)
(PCB) technology, while still provide accurate phase shift in relatively wide bandwidth (usually $>50 \%$ ), making them stand out from various wideband phase shifter designs.

The original Schiffman phase shifters are based on stripline structures [1]. When applying the design approach to the microstrip circuits, the uneven phase velocity of odd and even modes of the coupling region leads to impedance mismatch and hence large insertion loss. Different approaches were discussed to solve this problem, including introducing the multi-section coupled lines [4], adopting advanced analysis method which calculates the actual phase velocities [6] and removing part of the ground to reduce the even mode phase velocity [7]. Analytical equations for approximate calculations were presented in these works, which can be used to guide the design. However, due to the approximations being used and the unquantifiable influences of different structures (e.g. chamfered entries, fringing effects and parasite inductances of the narrow links between coupled lines), it's hard to analytically calculate the electromagnetic (EM) response of a Schiffman phase shifter given its dimensions. As a result, time-consuming fine-tuning is always required in the design process.

In recent years, Machine Learning (ML) techniques and Deep Neural Networks (DNNs) have been widely adopted in various fields such as computer vision (CV), natural language processing (NLP), computational learning. They have surpassed human performance in terms of both accuracy and efficiency in different tasks including self-driving systems, efficient web search and optical character recognition. Moreover, MLs and DNNs are well suited to solve the EM forward modeling and inverse design problems, due to their ability to map the highly nonlinear relationships between the inputs (physical quantities) and outputs (EM fields or responses). Existing works have successfully demonstrated the feasibility to employing different DNNs to address the forward characterization and inverse design of metamaterials $[25,26]$, metasurfaces [27-29] and microwave antennas [30, 31]. On the other hand, until very recently, the application of ML and DNNs in microwave circuits and other microwave components

B. Zheng, H. Tang, H. Li, L. Zhou, Y. Dong, M. Haerinia, and H. Zhang are with the Electrical and Computer Engineering Department, University of Massachusetts Lowell, Lowell, MA 01854 USA (e-mail: hualiang_zhang@uml.edu). 
is still a nascent field. Only a few works have discussed the implementation of DNNs for the modeling, optimization or inverse design of microwave components and circuits such as transitions [32], ground via [33], amplifiers [34], transmission line [35] and filters [36-44]. Among these works, Watson et al presented a knowledge-based artificial neural network (ANN) approach for the modeling of microstrip grounding via, coplanar waveguide (CPW) structures and stripline-to-stripline transitions [32, 33]. Zhang et al proposed an ANN-based CAD (computer-aided design) approach for the modeling and optimizations of FET, CPW filters and patch antennas [34, 43, 44]. Delgado et al presented a SYNTHESIS-ANN for the modeling of structures including microstrip line and printed antennas with balun [35]. In [36-41] Jin, Na and Feng et al proposed a DNN approach for the modeling of multivariate microwave components and the parameter extraction of microwave filters. The presented approach has been applied to predict the $S$-parameters of different types of microwave filters and extract the coupling matrices of given $S$-parameter targets. In [42] Zhang et al proposed an ANN approach for the optimization of a bandstop microstrip filter. With the DNNs and ANNs adopted in these works, conventional time-consuming fullwave simulation and parametric modeling process can be substituted (and carried out) with a well-trained network on a one-time calculation basis, and thus greatly reducing the overall modeling and design time.

In this paper, we show how a DNN based on concise fullyconnected architecture can be utilized for the fast forward modeling and inverse design of microwave components. Due to the capability of mapping the highly nonlinear relationships between inputs and outputs, the DNNs are capable of predicting accurate phase and amplitude responses of an EM component given its design parameters. Once fully-trained, the DNNs can generate prediction results in milliseconds, and thus enabling fast on-demand designs. Without loss of generality, a class of microstrip Schiffman phase shifters are explored for the purpose of demonstration. For the first time, a predicting neural network that is capable of simultaneously modeling the phase shift, insertion loss and return loss of Schiffman phase shifters over a relatively wide spectrum (133\%) has been demonstrated. Based on the highly accurate forward predicting network, a tandem inverse design network was constructed for the fast ondemand designs of Schiffman phase shifter with arbitrary phase shift and bandwidth targets. To verify the efficacy of the proposed approach, two groups of phase shifter designs, with $60 \%$ and $40 \%$ bandwidth respectively, were designed, fabricated and measured. The good agreement between design targets and measurement results substantiate that the proposed approach accomplished two important goals in the field of EM component design: 1) building a fast and accurate simulation tool to validate the performance of EM components and 2) generating the layout of on-demand designs instantly, with no further optimization and fine-tuning needed. It is expected that the proposed approach can be easily generalized to other EM problems, including but not limited to antenna design, microwave circuit design and EM compatibility problems. (a)

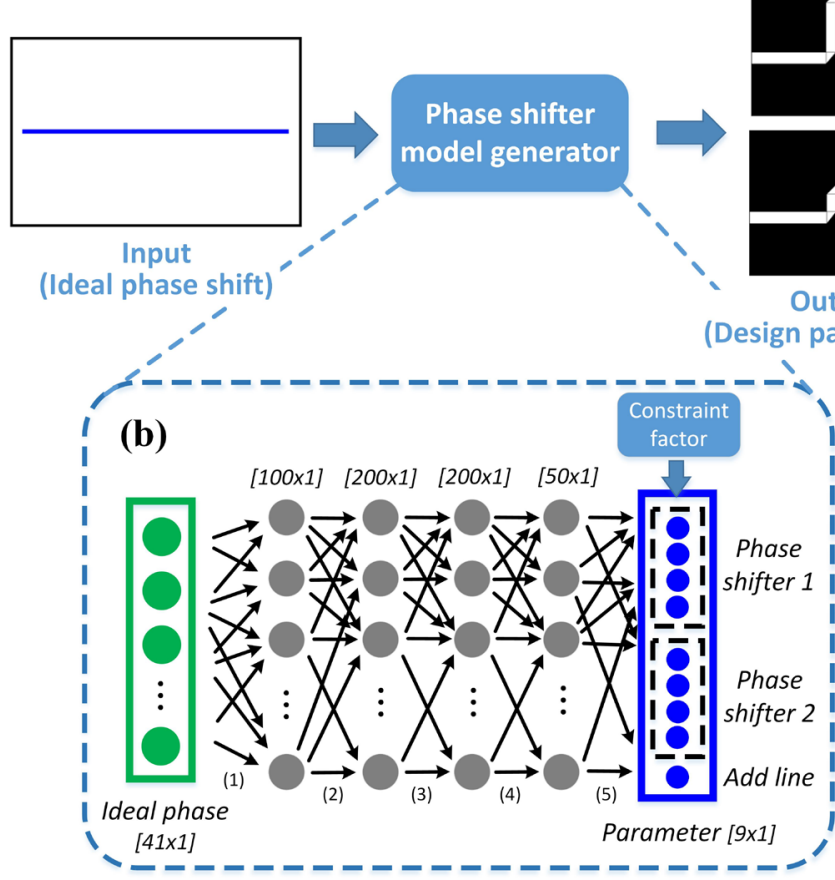

(c)
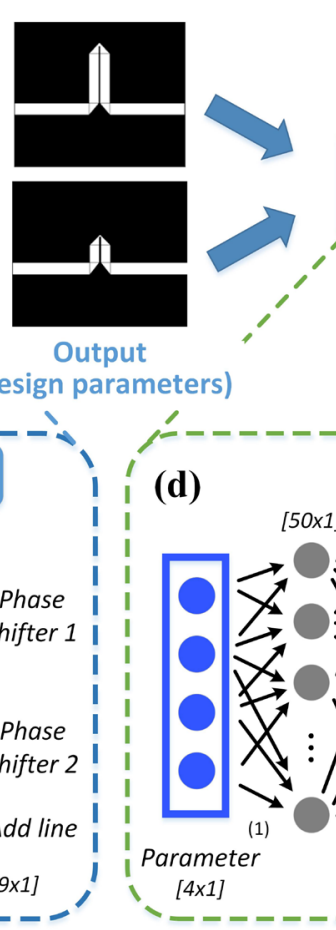

(e)

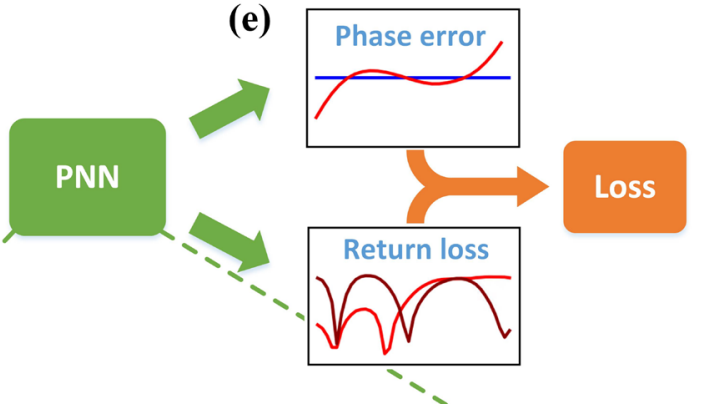

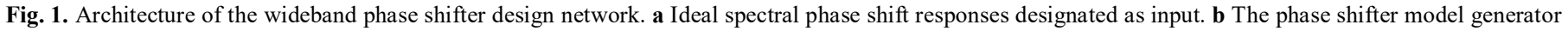

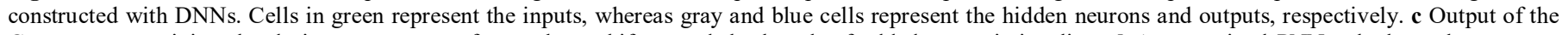

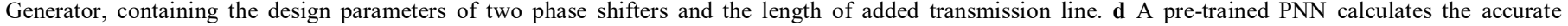

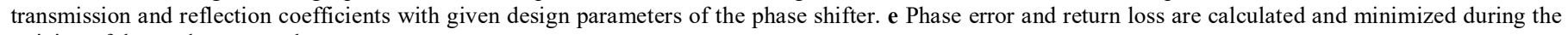
training of the tandem network. 


\section{NETWORK STRUCTURE}

The proposed wideband phase shifter design network is illustrated in Fig. 1. The design goal is to generate differential phase shifters with user-defined phase profile while maintaining low return loss and insertion loss. Since it is possible to have multiple different devices with similar EM responses, constructing a simple inverse design network with target responses assigned as input and design parameters as output will cause the network unable to converge [27, 36], due to the conflicting input-output data pairs in the design pool. To address this problem, a "tandem" network architecture [26, 27] is adopted, which consists of an inverse design model generator (hereinafter called the 'Generator') and a predicting neural network (hereinafter called the 'PNN') to form a cascaded architecture. The input and output for a "tandem" network are both EM responses, which eliminates the possibilities of nonunique solutions and thus stabilizes the training process. As shown in Fig. 1a, the ideal spectral phase response is discretized into a $1 \mathrm{D}$ vector, with all data points evenly distributed in the spectrum of interest. The Generator in Fig. 1b consists of four consecutive fully-connected hidden layers each containing 100 , 200, 200 and 50 hidden neurons, respectively. The Generator takes the ideal phase shift as input and generates the design parameters of two phase shifters (Fig. 1c), which produce the on-demand differential phase shift. The design parameters are then designated as input for a well-trained PNN (Fig. 1d), where the two phase shifters' transmission and reflection coefficients are evaluated when passed through four full-connected hidden layers consisting of 50, 500, 500 and 200 hidden neurons, respectively. Finally, the network calculates a combined loss function (Fig. 1e), which is a weighted sum of the phase error and the return loss. During the training, the weights and biases in the hidden layers of the Generator are optimized to minimize this loss, while the values of hidden neurons in the previouslytrained PNN remain unchanged. As a result, the Generator becomes "smarter" as training proceeds, eventually forming a cascaded DNN capable of generating on-demand Schiffman phase shifter designs on a one-time calculation basis.

\section{A. Forward Prediction}

In order to enable the inverse design, a PNN (as shown in Fig. 1d) is trained first to realize the fast and accurate evaluation of Schiffman phase shifters. The PNN functions as a powerful alternative to the full-wave simulation tool. It is considered as the key building block in the tandem network structure for two reasons: 1) the Generator relies on the PNN to distinguish the bad designs from the good ones and thus help the Generator choose the right gradient descend direction and become "smarter" in design during the training. An inaccurate PNN will eventually lead to a defective Generator even after fully trained. 2) During the training of the Generator, each generated phase shifter design needs to be quickly evaluated to derive the training loss. Thousands of designs are generated and evaluated during one training iteration, which is normally done in seconds. This time efficiency is only possible with a fully trained PNN.
As shown in Fig. 2a, four design parameters including the length and width of the coupled microstrip lines and the connection section, were used to parameterize the Schiffman phase shifter structures. Without loss of generality, we chose Rogers $\mathrm{R} 4003 \mathrm{C}\left(\varepsilon_{r}=3.55, \tan \delta=0.0027\right)$ with the thickness of $1.524 \mathrm{~mm}$ as the substrate. The spectra of interest are set to be from 1 to $5 \mathrm{GHz}$ with center frequency at $3 \mathrm{GHz}$. To train the PNN, a dataset containing over 53,000 random Schiffman phase shifters, along with their transmission and reflection coefficients was created using commercial software package CST Microwave Studio. Among them, 70\% were assigned to be the training set, while the remaining $30 \%$ were used as the test set. The whole spectrum is down-sampled into 41 frequency points (between 1 to $5 \mathrm{GHz}$ ) with a frequency step of $0.1 \mathrm{GHz}$, corresponding to 41 coefficients which are specified as the network output. To reduce the training complexity, the transmission and reflection coefficients are combined into the output of the PNN, which were predicted at the same time. Two independent neural networks were constructed to predict the real and imaginary parts of the coefficients, respectively. The loss functions we used for PNNs are:

$$
L_{P N N}=\frac{1}{N} \sum_{i=1,2 \ldots N}\left(S_{P N N}-S_{t r u t h}\right)^{2}
$$

where $S$ is the spectral responses. After fully trained, the test set error of the real and imaginary PNNs are $1.6 \times 10^{-5}$ and $1.3 \times$ $10^{-5}$, equivalent to a phase error of 0.1 degree and amplitude error of 0.005 at a single frequency point. Several prediction samples were randomly selected from the test dataset and showcased in Fig. 2, while more prediction samples are presented in the Appendix Fig. A2. These examples show excellent consistency between numerical simulations and PNN predicted results across the full spectrum. Due to its one-time calculation nature, the well-trained PNNs are capable of modeling the performances of different Schiffman phase shifters with almost no time cost (e.g. in milliseconds), which enables the fast inverse design when cascaded with optimization algorithms or DNNs (as will be discussed in the next section).
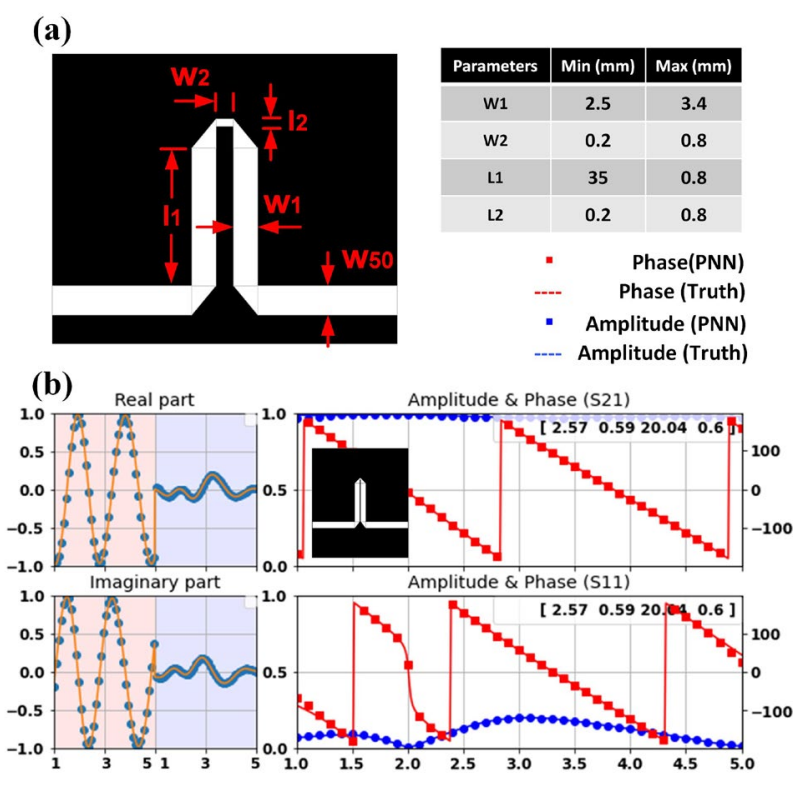

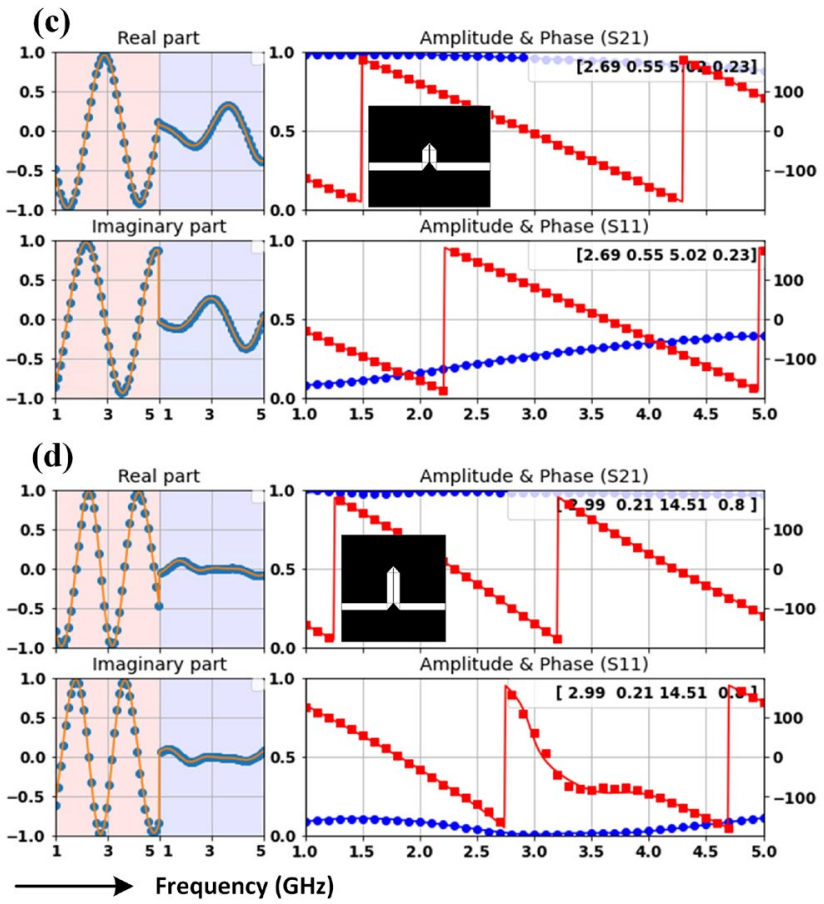

Fig. 2. PNN for Schiffman phase shifters. a Top-view figure showing the dimensions that parameterize the Schiffman phase shifter structure. b-d Examples of EM responses of Schiffman phase shifters predicted by the PNN (dots) and full-wave numerical simulations (lines). The real and imaginary part of the predicted complex transmission coefficients are shown on the left in each subplot, with reflection coefficients highlighted in orange and transmission coefficients highlighted in cyan. Design parameters are given as insets in the following order: $W_{l}, W_{2}, L_{1}$ and $L_{2}$ (in mm). $W_{50}$ denotes the width of the 50Ohm transmission line.

\section{B. Inverse Design}

To demonstrate the versatility of the proposed approach, two Generators that are capable of designing Schiffman phase shifters with two different pre-assigned fractional bandwidths $(40 \%$ and $60 \%)$ were trained. The same dataset was used during these two training processes, which contains randomlygenerated ideal flat phase shift targets ranging from 0 to 180 degrees. Since the inputs are not labeled with corresponding design parameters, the training was executed in an unsupervised way. The output layer contains 9 values, including the design parameters of two differential phase shifters and the length of an extra 50-Ohm transmission line cascaded to the first phase shifter which is used to adjust the phase shift value. With the help of the well-trained PNN, the insertion loss, phase and return loss of both phase shifters, along with the differential phase shift between them can be instantly calculated. The loss functions of the cascaded network can be expressed as the weighted sum of three least square errors, including the phase error of current design, distance from current design to preset parameter value range and the return loss, which are derived as:

$$
\begin{aligned}
& L_{\text {Generator }} \\
& =\frac{1}{N} \sum \begin{array}{c}
A \cdot\left(S_{P N N}-S_{\text {target }}\right)^{2}+ \\
B \cdot\left(P-P_{\text {clipped }}(\text { max }, \text { min })\right)^{2}+ \\
C \cdot \operatorname{ReLU}\left(S_{11}-\text { threshold }\right)
\end{array}
\end{aligned}
$$

where $A, B$ and $C$ are the weight coefficients, $P$ is the output vector (Fig. 1c) containing design parameters and $P_{\text {clipped }}$ is a vector with all values in $P$ clipped to a preset maximum and minimum value (Fig. 2a). The constraint factor (second term in equation 2) measures the distance from output to the desired parameter value range. When the vector $P$ falls out of the preset value range, the increasing loss function value will force the optimizer to regress to generate a $P$ value within the desired range (max, $\min$ ). When the return loss of the generated design is higher than the preset threshold (set to be $-14 \mathrm{~dB}$ during training), the third term in equation 2 will generate an error larger than zero and force the optimizer to generate a different design with lower return loss. Since the second and third terms in equation 2 should be equal to zero with an ideal phase shifter design, their corresponding weight coefficient is set to be a much larger value $(A=1, B=C=500)$. After 1,000 epochs of training for each group of data, the combined error eventually stabilized at 0.84 and 7.85 for $40 \%$ bandwidth targets and $60 \%$ bandwidth targets, respectively (details can be found in the Appendix Fig. A1 and Table I). The larger error for $60 \%$ bandwidth targets manifests the increasingly design difficulty of Schiffman phase shifters with a larger bandwidth. Fig. 3 shows four representative design examples, including two 90degree and two 180-degree phase shifters designed by both Generators. The relatively low phase error $\left(90^{\circ} \pm 1.6^{\circ}, 180^{\circ} \pm 2^{\circ}\right.$ designed by the $40 \%$ bandwidth Generator, $90^{\circ} \pm 3^{\circ}, 180^{\circ} \pm 12^{\circ}$ designed by the $60 \%$ bandwidth Generator) indicates the good efficacy of the proposed deep learning approach. (a)

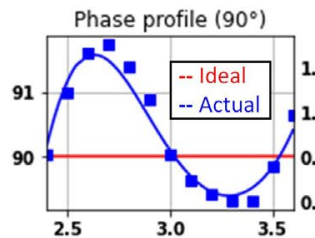

(b)

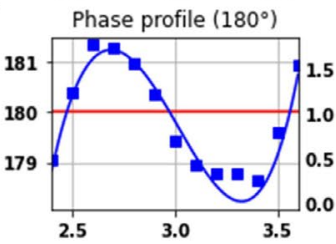

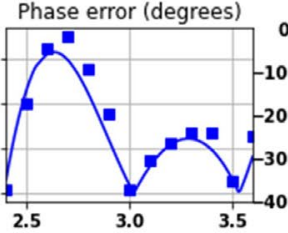

Phase error (degrees)

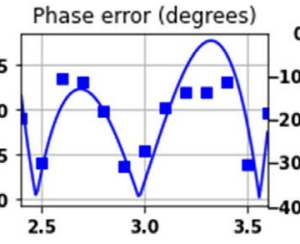

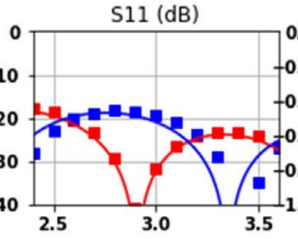

$\mathrm{S} 11(\mathrm{~dB})$

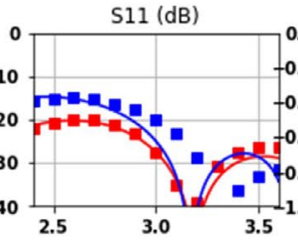

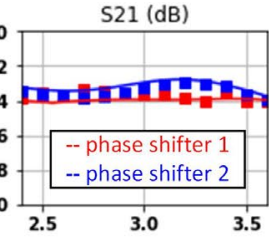

$\mathrm{S} 21(\mathrm{~dB})$

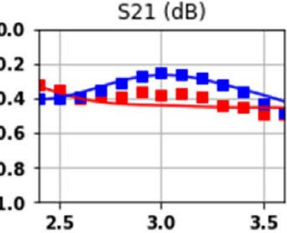

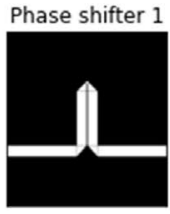

Phase shifter 1

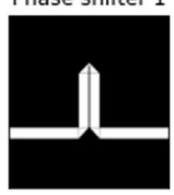

Phase shifter 2

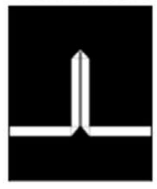

Phase shifter

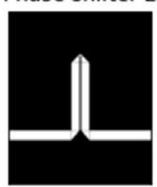


(c)
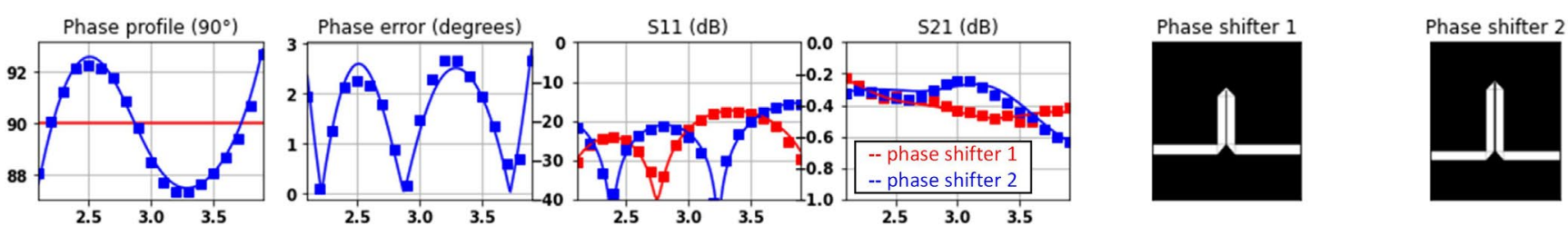

(d)
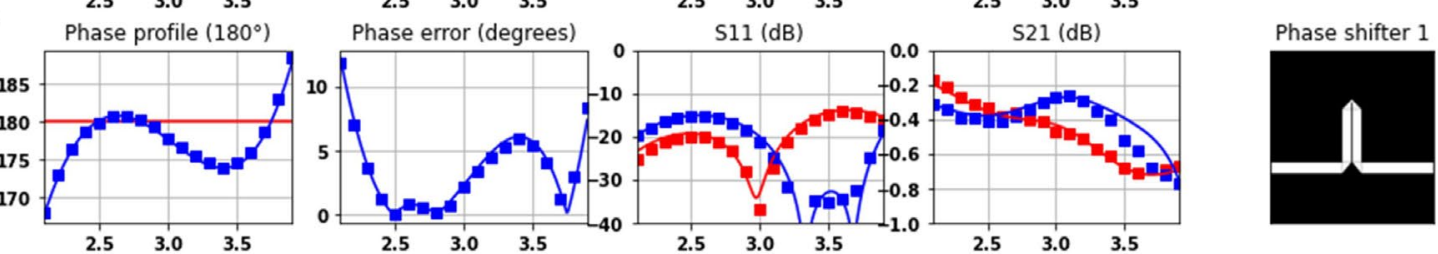

Phase shifter 2

Fig. 3. Phase shifter examples designed by the well-trained Generators. a 90 degree, $40 \%$ bandwidth. b 180 degree, $40 \%$ bandwidth. c 90 degree, $60 \%$ bandwidth.

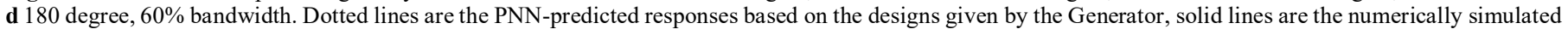
responses.

As mentioned earlier, the highly-efficient nature of this DNN approach allows us to generate high-performance phase-shifter designs with almost no time cost. To demonstrate this unique feature, as showcased in Fig. 4, 36 phase shifter designs with phase shift targets ranging from 5 degrees to 180 degrees (with 5-degree interval) were designed using the $40 \%$ bandwidth and $60 \%$ bandwidth Generators, respectively. The corresponding differential phase shifts were evaluated with the PNNs. It's worth to mention that all designs in Fig. $4 \mathrm{a}$ and Fig. $4 \mathrm{~b}$ were generated and characterized within milliseconds.
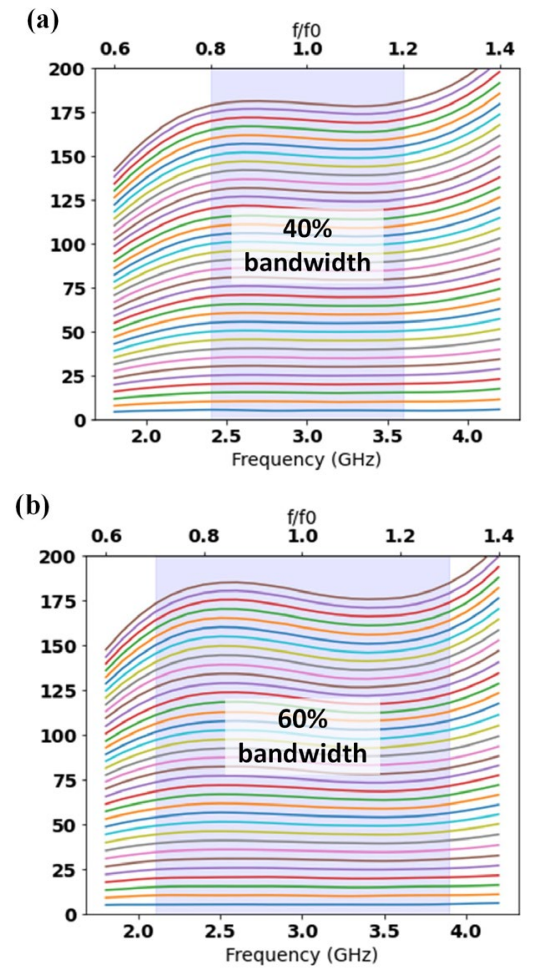

Fig. 4. More phase shifter designs by the well-trained Generators. Performance of 36 Schiffman phase shifters with design targets ranging from 5 degrees to 180 degrees (5-degree interval). a designs by the $40 \%$ bandwidth Generator. b Designs by the $60 \%$ bandwidth Generator.

\section{EXPERIMENTAL VERIFICATION}

In order to verify the proposed method, two 90-degree differential phase shifters presented in Fig. $3 a$ and $3 c$ were fabricated and measured. Detailed parameters of these two designs are given in the Appendix Table II. The substrate for the microstrip line is Rogers RO4003C with the dielectric constant of 3.55 , to keep consistency with the setups used during the data collection process. The measured return loss,
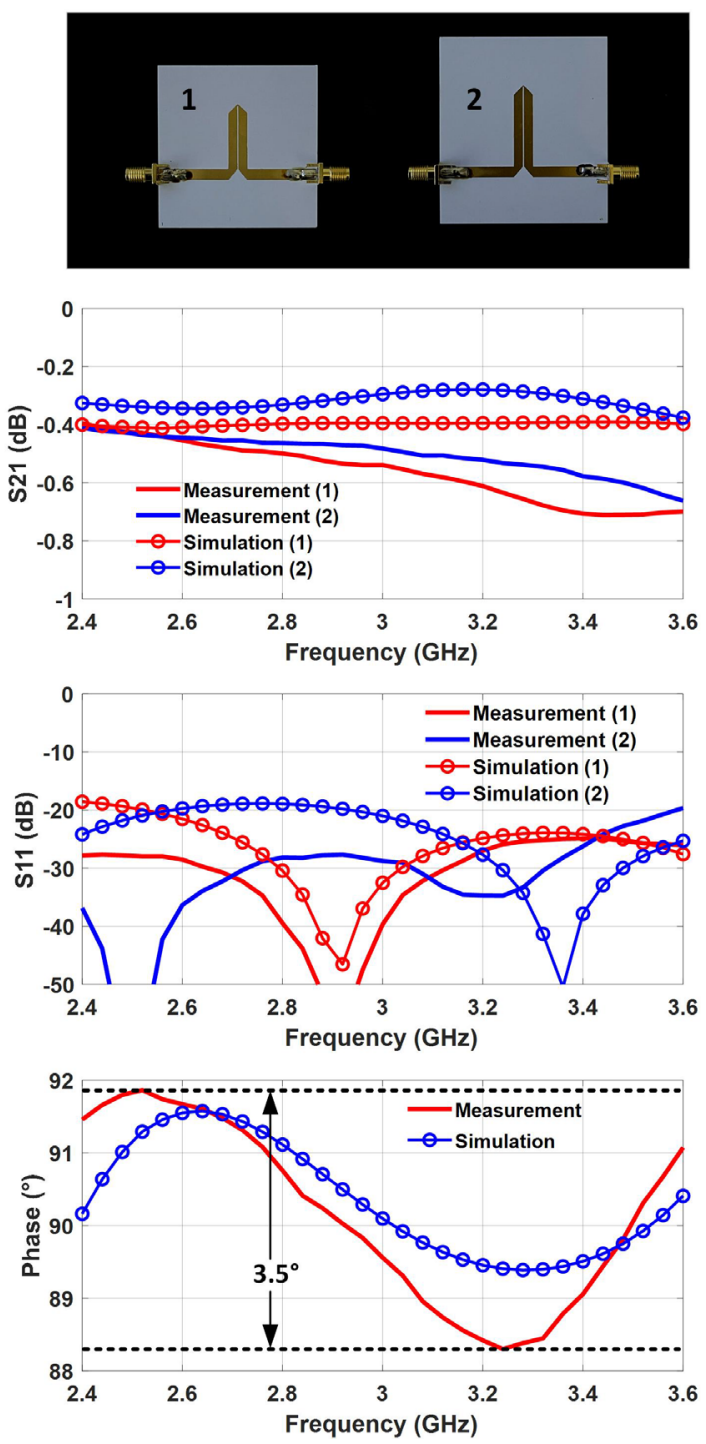

Fig. 5. Measurement results of the 90 -degree Schiffman phase shifters with $40 \%$ fractional bandwidth 

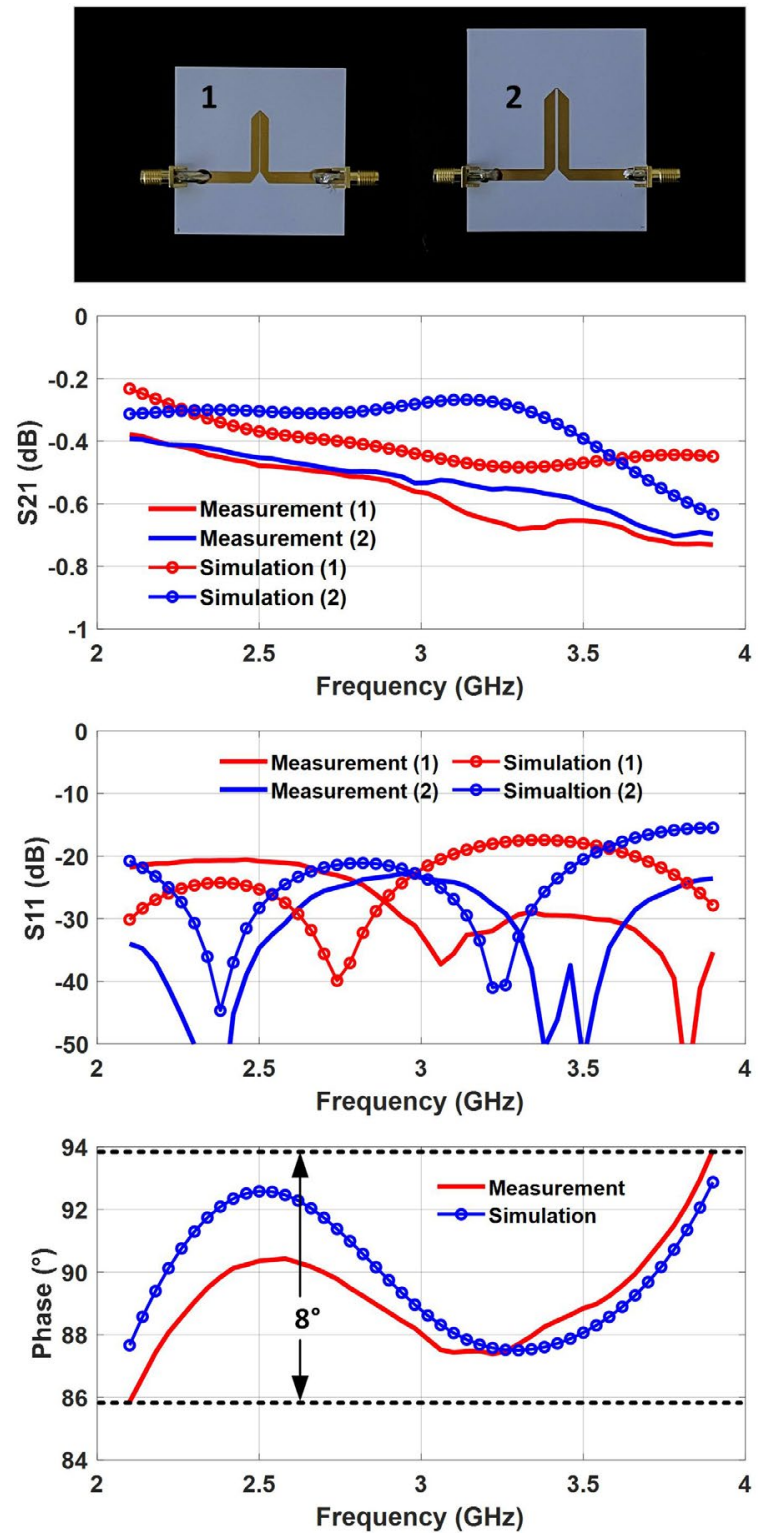

Fig. 6. Measurement results of the 90-degree Schiffman phase shifters with $60 \%$ fractional bandwidth.

insertion loss and differential phase shift of the two phase shifters are presented in the subplots of Figs. 5-6. The recorded minimum return loss, maximum insertion loss and maximum phase error are $19.64 \mathrm{~dB}, 0.71 \mathrm{~dB}$ and $\pm 1.86^{\circ}$ for the $40 \%$ bandwidth $(2.4 \mathrm{GHz}-3.6 \mathrm{GHz})$ phase shifter and $20.68 \mathrm{~dB}$, $0.73 \mathrm{~dB}$ and $\pm 4.1^{\circ}$ for the $60 \%$ bandwidth $(2.1 \mathrm{GHz}-3.9 \mathrm{GHz})$ phase shifter. As shown in Figs. 5-6, the full-wave simulation results are also included as reference. The measured results are in reasonably good agreement with the simulation results, which validates both the accuracy of the pre-trained PNN and the efficacy of this inverse design approach.

\section{FURTHER DISCUSSION}

This deep learning approach shows its unique advantages over conventional methods when dealing with designs with more degrees of freedom. As discussed in the literature, the phase errors in microstrip Schiffman phase shifters caused by the uneven phase velocity of odd and even modes can be moderated by applying measures such as introducing multisection coupled lines [4], adopting advanced analysis method [6] and modifying the ground plane [7], which all resulted in complex architectures that are extremely hard (if not impossible) to model with traditional field and circuit analytical solutions. Therefore, these improved designs are derived with either particular solution based on presumptions that limited the design degrees of freedom [4], or trial-and-error method which requires time-consuming fine-tuning process [7]. In contrast, the proposed prediction and inverse design process with DNNs are data-driven. With hidden-layers that provide sufficient hidden units, the presented DNNs are able to uncover the highly nonlinear hidden relations between inputs and outputs, even the complicated ones that are unable to be modeled with analytical equations, according to the universal approximation theorem $[45,46]$. As a result, given sufficient amount of training data, PNNs with similar network structures can be used to model different types of structures, regardless of their complexities. To explore the proposed approach's potential in dealing with more complicated geometries, we trained another PNN and Generator for the forward modeling and inverse design of a dual-section Schiffman phase shifter. As shown in Fig. 7a, the phase shifter with dual-section coupled line structure can be parameterized into seven parameters, including the length, width of the coupled lines and the gap between them. The network architecture in Fig. 1 was slightly modified for the training of the PNN and the model Generator for dual-section Schiffman phase shifters. Specifically, we change the output size of the Generator to 15 by 1 and the input size of the PNNs to 7 by 1 . Over 60,000 groups of training data were then collected with the parameters randomly generated within the preset value range in Fig. 7a. After fully trained, the combined error finally stabilized at the value of 1.98 with the same loss function in equation 2. Compared with the single-section Generator with the final error of 7.85, this significantly lower error value clearly indicates that Schiffman phase shifters with better performance can be achieved with this dual-section structure. We then used the well-trained dual-section Generator to design a 180-degree phase shifter with $60 \%$ fractional bandwidth (design details are included in the Appendix Table III). The design diagram, phase, return loss and insertion loss of the proposed design are shown in Fig. $7 \mathrm{~b}-\mathrm{d}$, respectively. Compared with the 180-degree phase shifter design in Fig. 3d, this dual-section design was able to reduce the phase error by $60 \%$ while maintaining similar level of return loss and insertion loss, which clearly indicates the advantage of introducing more design degrees of freedom.

A major concern for this data-driven design approach is the time-consuming data collection process. Fortunately, unlike other regression or classification problems in the deep learning field, the data in EM design problems is cheap and easilyaccessible, with the majority of the cost coming from electricity consumption. It's also worth noting that the data collections in this paper are done in an unsupervised way, and this process can be easily accelerated by running the simulations in parallel. For example, it took 3 and 4 days to collect enough amount of 
training data for the PNNs in Fig. 2 and Fig. 7 with an 8-node cluster, respectively.

(a)

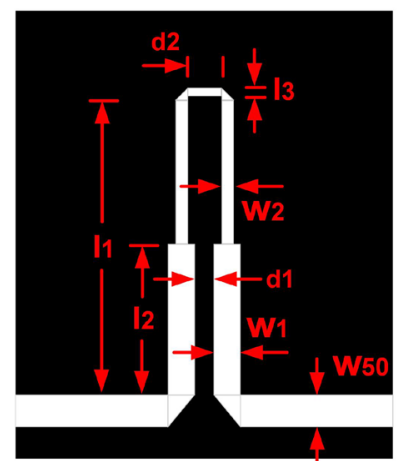

\begin{tabular}{|c|c|c|}
\hline Parameters & $\operatorname{Min}(\mathrm{mm})$ & $\operatorname{Max}(\mathrm{mm})$ \\
\hline W1 & 2.5 & 3.4 \\
\hline L1 & 6 & 35 \\
\hline W2 & 0.2 & 0.8 \\
\hline L2 & 2 & L1-2 \\
\hline L3 & 0.2 & 0.8 \\
\hline D1 & 0.2 & 0.8 \\
\hline D2 & 0.2 & 0.8 \\
\hline
\end{tabular}

(b)
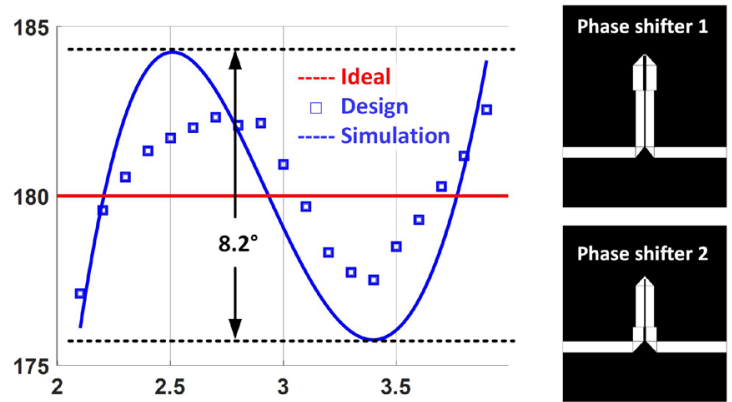

(c)

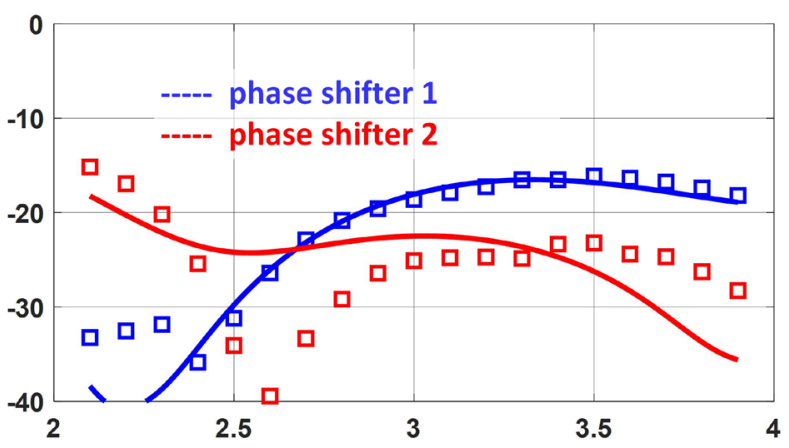

(d)

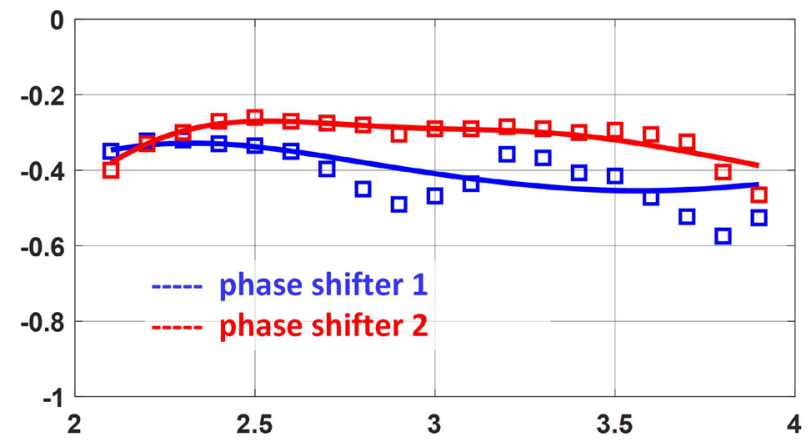

Fig. 7. A 180-degree dual-section Schiffman phase shifter design with $60 \%$ fractional bandwidth. a Top-view figure showing the dimensions that parameterize the dual-section Schiffman phase shifter structure. b Diagram of the designs and corresponding phase shift. c Return loss. d Insertion loss. Dotted lines are the PNN-predicted responses based on the designs given by the Generator, solid lines are the numerically simulated responses.

\section{CONCLUSION}

To conclude, we have proposed a novel DNN-based approach for the inverse design of high-performance Schiffman phase shifters. Based on the tandem DNN architecture, we have demonstrated the fast and accurate forward characterization and inverse design of a class of Schiffman phase shifters. Several design examples, including two 90-degree phase shifters and two 180-degree phase shifter with $40 \%$ and $60 \%$ fractional bandwidth were designed and numerically verified. The results are comparable to the state-of-the-art designs [7, 11, 12].

This deep learning approach not only quickly explores the locally optimal designs for given structures, but also eliminates the fine-tuning process that was increasingly time-consuming in structures with complicated shapes. Different from traditional design approaches or local optimization algorithms, the presented DNNs process the input data in parallel, thus the training and design time of the PNNs and Generators are not affected by the number of parameters, making them suitable for the inverse design of multi-parameter problems. Although this paper mainly discusses the forward modeling and inverse design of Schiffman phase shifters operating from 1 to $5 \mathrm{GHz}$ spectrum, the deep learning approach for objective-driven design developed herein is not limited to specific bandwidths, phase shifter types or device categories. The proposed framework can be adapted to the design of various electromagnetic components that can be sketched with several design parameters, including but not limited to antennas, filters, power dividers, and frequency selective surfaces (FSS). Moreover, by adopting the convolutional neural network (CNN) layers, the PNN can deal with image input [28], which further increases its adaptability.

\section{APPENDIX}

In the Appendix, we provide further details on network modeling, network training and additional examples showcasing the performances of different networks. This Appendix comprises the following Sections:

\section{A. Hyperparameters used during DNN training process}

Hyperparameters used in the training for both PNNs and all three Generators are shown in Table I. The hardware consists of a quad-core CPU with $3.5 \mathrm{GHz}$ clock speed, 64 Gigabytes of RAM and two NVidia 1080Ti GPUs. As shown in the table, after 20,000 iterations, the average test set error for single section PNNs stabilized at 0.000016 and 0.000013 for the real and imaginary parts, respectively. The final test error for dual section PNNs are 0.000098 for the real parts and 0.00012 for the imaginary parts, respectively. With the current hardware setup, the training takes 4 hours for all four PNNs before their error rates stabilize. The model Generator networks, on the other hand, takes less training time due to the smaller network dimensions and less non-intuitive inverse design rules. All three Generators are well-trained after 1,000 iterations, each requiring less than 20 minutes. 


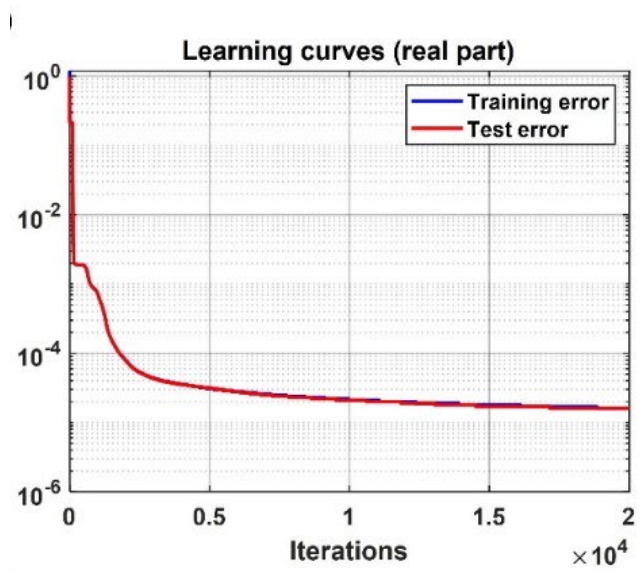

(b)
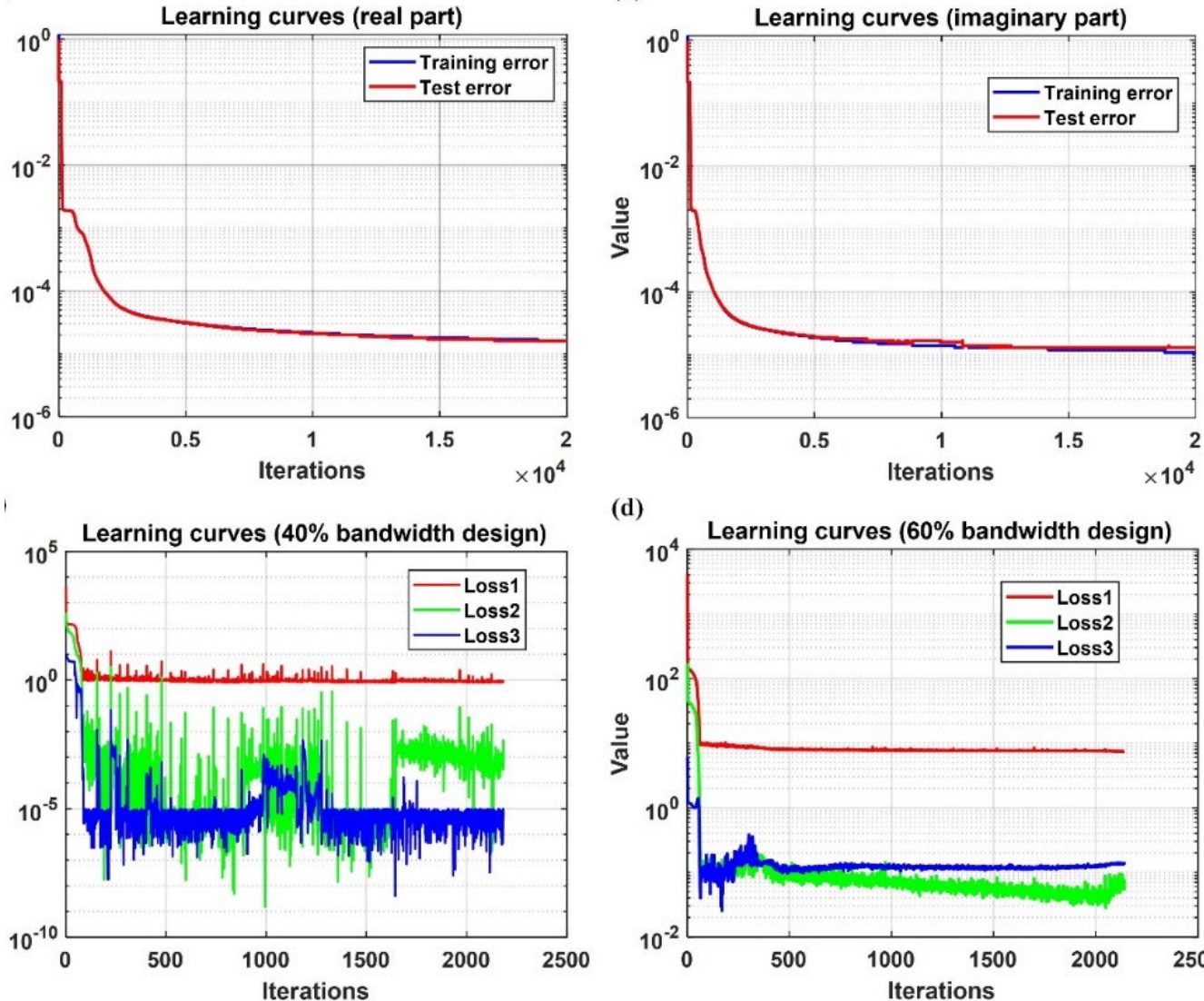

(d)
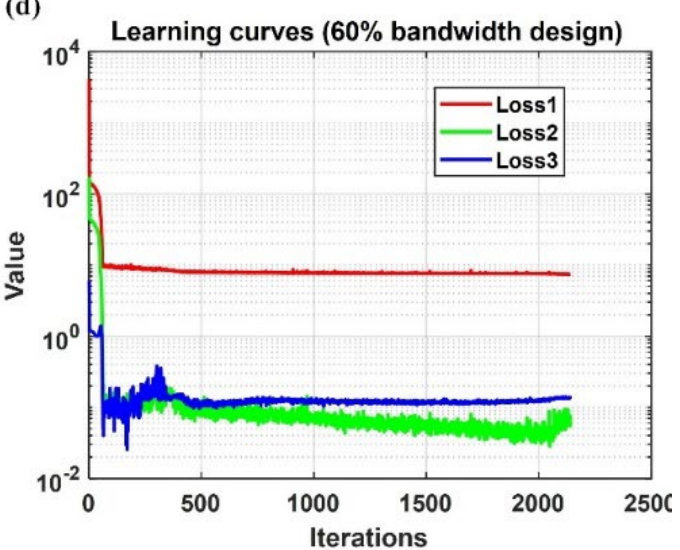

Fig. A1. Learning curves of the PNNs \& model Generators. Shown in the figures are the learning curves of a real part PNN, $\mathbf{b}$ imaginary part PNN, $\mathbf{c}$ Phase shifter Generator with $50 \%$ fractional bandwidth and $\mathbf{d}$ Phase shifter Generator with $60 \%$ fractional bandwidth. Loss 1, 2 and 3 corresponds to the first, second and third terms in Eqn. 2, respectively.

TABLE I

HYPERPARAMETERS USED DURING THE TRAINING OF PNN AND PHASE SHIFTER GENERATOR

\begin{tabular}{|c|c|c|c|c|c|}
\hline \multirow[b]{2}{*}{ Hyper-parameters } & \multicolumn{2}{|c|}{ PNN } & \multicolumn{3}{|c|}{ Phase shifter Generator } \\
\hline & Single section & Dual section & $\begin{array}{l}40 \% \text { Bandwidth, } \\
\text { Single section }\end{array}$ & $\begin{array}{l}60 \% \text { Bandwidth, } \\
\text { Single section }\end{array}$ & $\begin{array}{l}60 \% \text { Bandwidth, } \\
\text { Dual section }\end{array}$ \\
\hline Training set size & 37,100 & 42,000 & 1,000 & 1,000 & 1,000 \\
\hline Test set size & 15,900 & 18,000 & / & l & l \\
\hline Optimizer & Adam & Adam & & Adam & \\
\hline Learning rate & $10-5$ & $10-5$ & & $10-4$ & \\
\hline Batch size & 200 & 200 & & 200 & \\
\hline Batch Norm. & No & No & & No & \\
\hline Nonlinear activations & ReLU & ReLU & & ReLU & \\
\hline Iterations & 20,000 & 20,000 & 2,200 & 2,200 & 3,000 \\
\hline Time taken & $2 \mathrm{~h}$ & $2 \mathrm{~h}$ & $15 \min$ & $15 \mathrm{~min}$ & $17 \mathrm{~min}$ \\
\hline Error (train) & $\begin{array}{l}1.6 \mathrm{E}-5 \text { (real) } \\
1.1 \mathrm{E}-5 \text { (imag) }\end{array}$ & $\begin{array}{c}4.3 \mathrm{E}-5 \text { (real) } \\
3.2 \mathrm{E}-5 \text { (imag) }\end{array}$ & 0.84 & 7.85 & 1.98 \\
\hline Error (test) & $\begin{array}{c}1.6 \mathrm{E}-5(\mathrm{real}) \\
1.3 \mathrm{E}-5(\mathrm{imag})\end{array}$ & $\begin{array}{c}9.8 \mathrm{E}-5 \text { (real) } \\
1.2 \mathrm{E}-4(\mathrm{imag})\end{array}$ & / & / & / \\
\hline
\end{tabular}


B. Additional samples of the PNN for single-section Schiffman phase shifters

In each panel, the red curve represent the PNN predicted values. The blue curves depict numerically simulated values obtained with the commercial frequency domain solver (CST Microwave Studio). Reflection coefficients are highlighted in orange and transmission coefficients are highlighted in cyan. Randomly-generated design parameters, including $W_{l}, W_{2}, L_{l}$ and $L_{2}$ (in $\mathrm{mm}$ ) are shown at the top of each panel (in the above order).

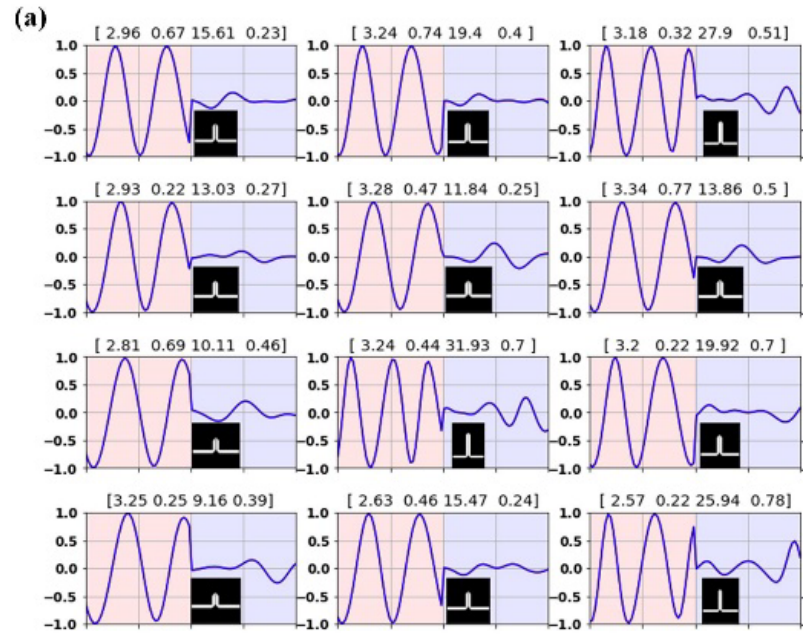

(b)

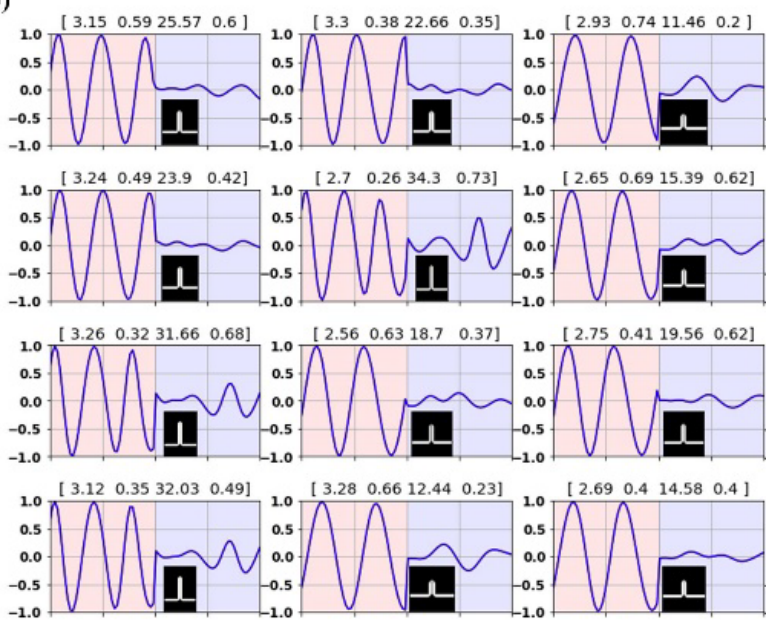

Fig. A2. Additional prediction examples generated with the single-section PNNs. a Real part prediction examples. b Imaginary part prediction examples.
C. Additional samples of the PNN for dual-section Schiffman phase shifters

In each panel, the red curve represent the PNN predicted values. The blue curves depict numerically simulated values obtained with the commercial frequency domain solver (CST Microwave Studio). Reflection coefficients are highlighted in orange and transmission coefficients are highlighted in cyan. Randomly-generated design parameters, including $W_{1}, W_{2}, L_{l}$, $L_{2}, L_{3}, D_{1}$ and $D_{2}$ (in $\mathrm{mm}$ ) are shown at the top of each panel (in the above order).
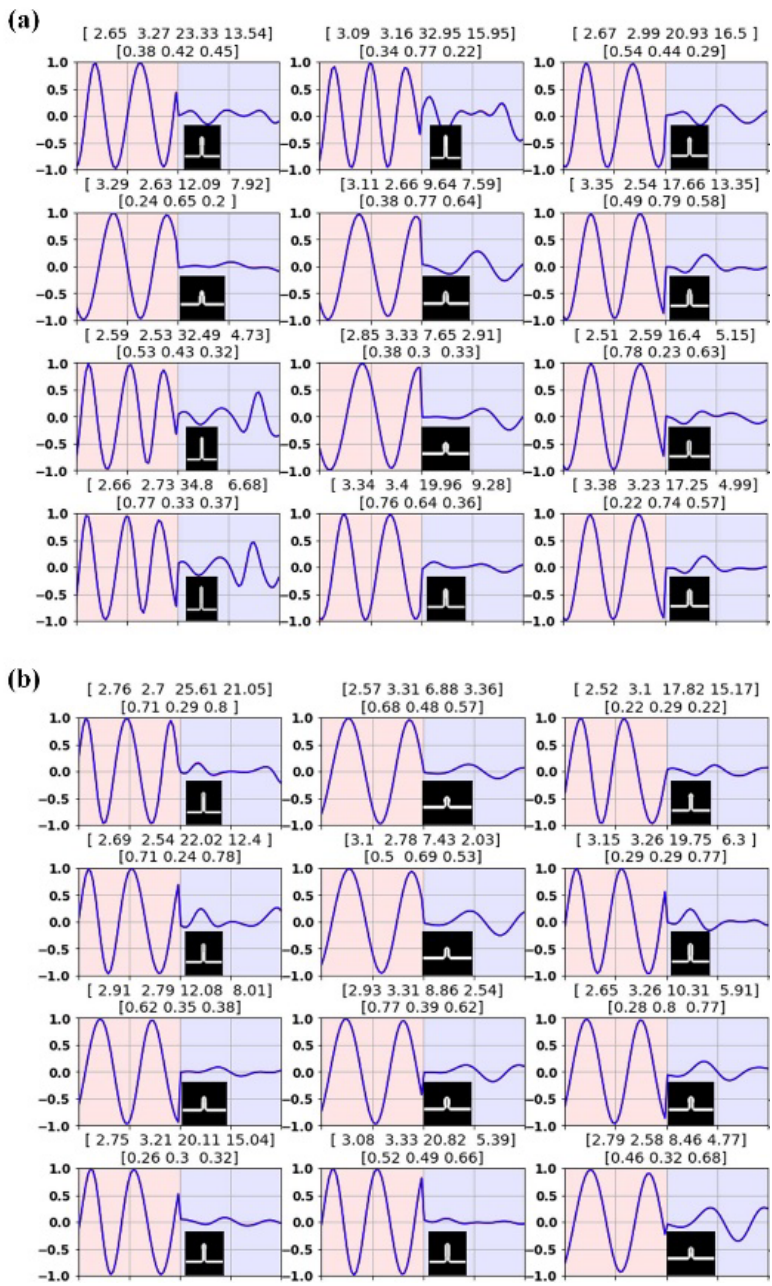

Fig. A3. Additional prediction examples generated with the dual-section PNNs. a Real part prediction examples. b Imaginary part prediction examples.

\section{Design details}

TABLE II

DETAILED DIMENSIONS OF THE PHASE SHIFTER DESIGNS IN FIG. 3

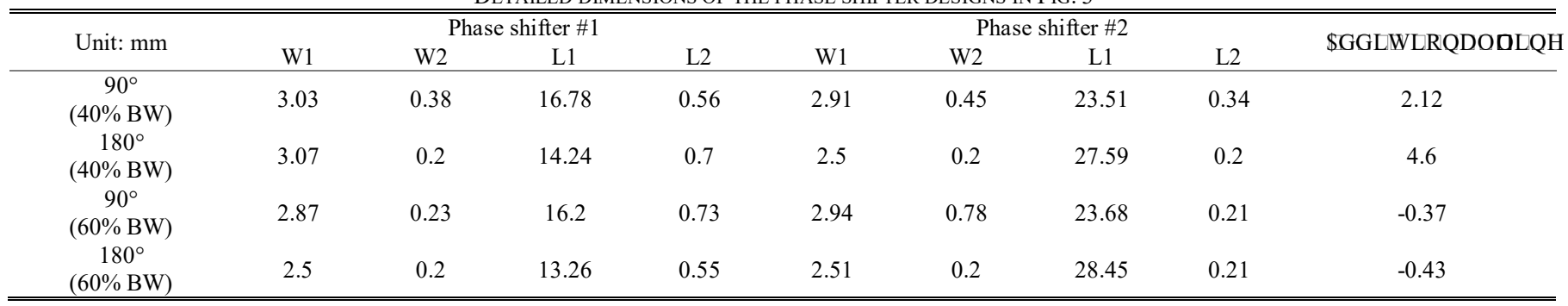


TABLE III

DETAILED DIMENSIONS OF THE PHASE SHIFTER DESIGNS IN FIG. 7

\begin{tabular}{|c|c|c|c|c|c|c|c|c|}
\hline \multirow[t]{2}{*}{ Unit: mm } & \multicolumn{7}{|c|}{ Phase shifter \#1 } & \multirow[t]{2}{*}{ \$GGLWLRQDOQLQH } \\
\hline & W1 & W2 & $\mathrm{L} 1$ & $\mathrm{~L} 2$ & L3 & D1 & D2 & \\
\hline \multirow{4}{*}{$\begin{array}{c}180^{\circ} \\
(60 \% \mathrm{BW})\end{array}$} & 3.21 & 2.8 & 16.91 & 4.35 & 0.68 & 0.78 & 0.2 & 9.08 \\
\hline & \multicolumn{7}{|c|}{ Phase shifter \#2 } & \\
\hline & W1 & $\mathrm{W} 2$ & L1 & $\mathrm{L} 2$ & L3 & D1 & D2 & \\
\hline & 2.74 & 3.29 & 24.82 & 17.25 & 0.22 & 0.2 & 0.8 & \\
\hline
\end{tabular}

\section{E. Network deployment}

To better showcase the time efficiency and accuracy of the proposed method, we have developed the well-trained DNN model and built a Schiffman phase shifter inverse design system (Fig. A4) using MATLAB built-in App Designer. This inverse design system takes the target phase shift and desired fractional bandwidth as input, and generates the design schematic, design parameters as well as the predicted performances of generated designs as output. The workflow of this design system is as follows: 1) the target phase and fractional bandwidth input by the user is fed to the inverse design network, which generates the design parameters of two differential phase shifter structures (shown in the center box of Fig. A4) and 2) performance of these two structures including the $S_{11}, S_{21}$ and differential phase are calculated using the pre-trained PNNs and shown in the performance box on the bottom. The overall design time is displayed in the message box, which is normally milliseconds.

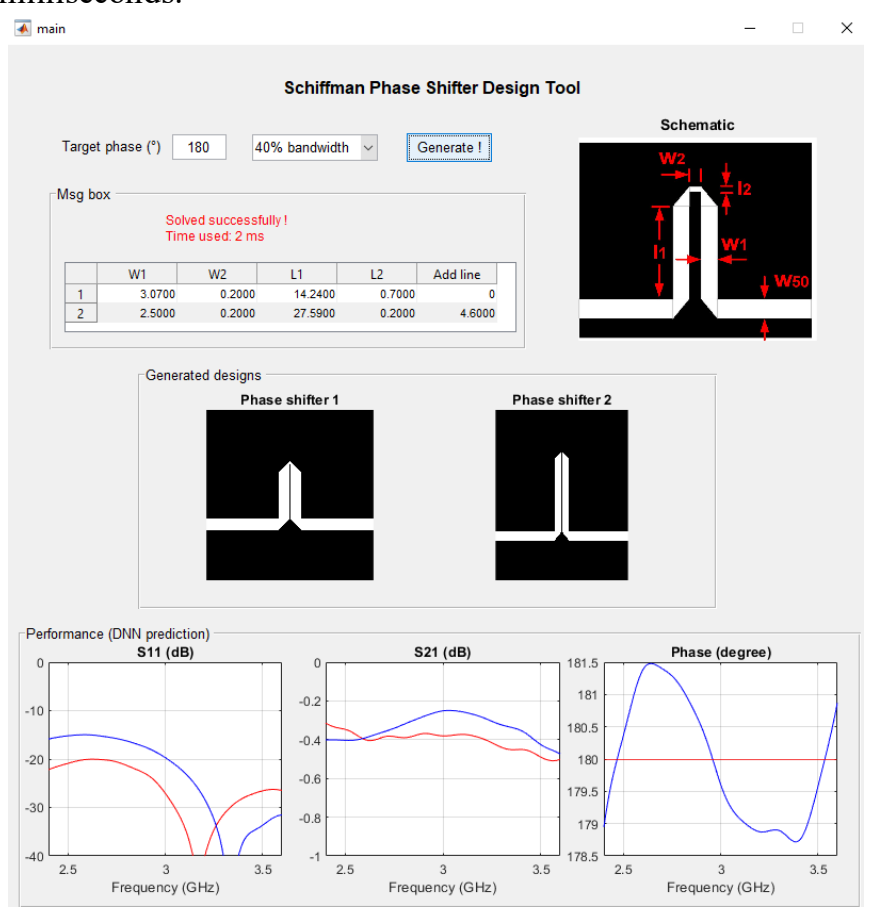

Fig. A4. User interface for the Schiffman phase shifter inverse design network. Target phase $\left(0\right.$ to $\left.180^{\circ}\right)$ and fractional bandwidth $(40 \%$ or $60 \%)$ are needed to generate the inverse phase shifter design. The design parameters, layout of the generated designs, along with the predicted performances are calculated simultaneously. A 180 -degree phase shifter with $40 \%$ fractional bandwidth was designed using this system as an example.

\section{ACKNOWLEDGMENT}

The datasets used during training and the deployed Schiffman phase shifter design tool is open source and available at: https://github.com/SensongAn/DNN-Schiffman-phaseshifter.

\section{REFERENCES}

[1] B. Schiffman, "A new class of broad-band microwave 90-degree phase shifters," IRE Transactions on Microwave Theory and Techniques, vol. 6, no. 2, pp. 232-237, 1958.

[2] Q. Liu, Y. Liu, J. Shen, S. Li, C. Yu, and Y. Lu, "Wideband Single-Layer $90^{\circ}$ Phase Shifter Using Stepped Impedance Open Stub and CoupledLine With Weak Coupling," IEEE Microwave and Wireless Components Letters, vol. 24, no. 3, pp. 176-178, 2014.

[3] Y. J. Cheng, K. Wu, and W. Hong, "Substrate integrated waveguide (SIW) broadband compensating phase shifter," in 2009 IEEE MTT-S International Microwave Symposium Digest, 2009: IEEE, pp. 845-848.

[4] B. Schiek and J. Kohler, "A method for broad-band matching of microstrip differential phase shifters," IEEE Transactions on Microwave Theory and Techniques, vol. 25, no. 8, pp. 666-671, 1977.

[5] J. R. Quirarte and J. P. Starski, "Novel Schiffman phase shifters," IEEE transactions on microwave theory and techniques, vol. 41, no. 1, pp. 914, 1993.

[6] C. E. Free and C. S. Aitchison, "Improved analysis and design of coupled-line phase shifters," IEEE Transactions on Microwave Theory and Techniques, vol. 43, no. 9, pp. 2126-2131, 1995.

[7] Y.-X. Guo, Z.-Y. Zhang, and L. C. Ong, "Improved wide-band Schiffman phase shifter," IEEE Transactions on Microwave Theory and Techniques, vol. 54, no. 3, pp. 1196-1200, 2006.

[8] Z. Zhang, Y.-C. Jiao, S.-F. Cao, X.-M. Wang, and F.-S. Zhang, "Modified broadband Schiffman phase shifter using dentate microstrip and patterned ground plane," Progress In Electromagnetics Research, vol. 24, pp. 9-16, 2011.

[9] W. Zhang, Y. a. Liu, Y. Wu, W. Wang, M. Su, and J. Gao, "A modified coupled-line Schiffman phase shifter with short reference line," Progress In Electromagnetics Research, vol. 54, pp. 17-27, 2014.

[10] S. E. Marini, J. Zbitou, R. Mandry, A. Errkik, A. Tajmouati, and M. Latrach, "Design of 45 degree microstrip phase shifter for beam forming network application using parallel coupled lines," in 2017 International Conference on Wireless Technologies, Embedded and Intelligent Systems (WITS), 2017: IEEE, pp. 1-3.

[11] Y.-P. Lyu, L. Zhu, and C.-H. Cheng, "Design and analysis of Schiffman phase shifter under operation of its second phase period," IEEE Transactions on Microwave Theory and Techniques, vol. 66, no. 7, pp. 3263-3269, 2018.

[12] L.-L. Qiu, L. Zhu, and Y.-P. Lyu, "Schiffman phase shifters with wide phase shift range under operation of first and second phase periods in a coupled line," IEEE Transactions on Microwave Theory and Techniques, vol. 68, no. 4, pp. 1423-1430, 2019.

[13] S. Zheng, S. H. Yeung, W. S. Chan, K. F. Man, and S. H. Leung, "Improved broadband dumb-bell-shaped phase shifter using multisection stubs," Electronics letters, vol. 44, no. 7, pp. 478-480, 2008.

[14] X. Tang and K. Mouthaan, "Design of a UWB phase shifter using shunt VWXEV LQ 2009 IEEE MTT-S International Microwave Symposium Digest, 2009: IEEE, pp. 1021-1024.

[15] S. Y. Zheng, W. S. Chan, and K. F. Man, "Broadband phase shifter using loaded transmission line," IEEE microwave and wireless components letters, vol. 20, no. 9, pp. 498-500, 2010. 
[16] S. H. Yeung, Z. Mei, T. K. Sarkar, and M. Salazar-Palma, "Design and testing of a single-layer microstrip ultrawideband 90 differential phase shifter," IEEE microwave and wireless components letters, vol. 23, no. 3, pp. 122-124, 2013.

[17] S. An, M. Badar, and Q. Zhu, "Generalized Analysis Method for a Class of Novel Wideband Loaded-Stub Phase Shifters," Radioengineering, vol. 24, no. 4, p. 927, 2015.

[18] B. Muneer, A. Sensong, A. W. Umrani, F. K. Shaikh, and U. Ahmed, "A generalized approach to analyze broadband arrow-shaped loaded-stub phase shifters."

[19] A. M. Abbosh, "Ultra-wideband phase shifters," IEEE Transactions on Microwave Theory and Techniques, vol. 55, no. 9, pp. 1935-1941, 2007.

[20] A. M. Abbosh, "Broadband fixed phase shifters," IEEE microwave and wireless components letters, vol. 21, no. 1, pp. 22-24, 2010.

[21] Y. Wang, M. Bialkowski, and A. Abbosh, "Double Microstrip-Slot Transitions for Broadband $\$\{\backslash \mathrm{pm}\} 90^{\wedge}\{\backslash \mathrm{circ}\} \$$ Microstrip Phase Shifters," IEEE microwave and wireless components letters, vol. 22, no. 2, pp. 58-60, 2012.

[22] Y. S. Wong, S. Y. Zheng, and W. S. Chan, "Multi-way and poly-phase aligned feed-forward differential phase shifters," IEEE transactions on microwave theory and techniques, vol. 62, no. 6, pp. 1312-1321, 2014.

[23] Y.-P. Lyu, L. Zhu, Q.-S. Wu, and C.-H. Cheng, "Proposal and synthesis design of wideband phase shifters on multimode resonator," IEEE Transactions on Microwave Theory and Techniques, vol. 64, no. 12, pp. 4211-4221, 2016.

[24] J. Zhou, H. J. Qian, and X. Luo, "Compact wideband phase shifter using microstrip self-coupled line and broadside-coupled microstrip/CPW for multiphase feed-network," IEEE Microwave and Wireless Components Letters, vol. 27, no. 9, pp. 791-793, 2017.

[25] S. So, J. Mun, and J. Rho, "Simultaneous Inverse-Design of Material and Structure via Deep-Learning: Demonstration of Dipole Resonance Engineering using Core-Shell Nanoparticles," ACS applied materials \& interfaces, 2019.

[26] D. Liu, Y. Tan, E. Khoram, and Z. Yu, "Training deep neural networks for the inverse design of nanophotonic structures," ACS Photonics, vol. 5, no. 4, pp. 1365-1369, 2018.

[27] S. An et al., "A Deep Learning Approach for Objective-Driven AllDielectric Metasurface Design," ACS Photonics, vol. 6, no. 12, pp. 3196 3207, 2019.

[28] S. An et al., "Deep learning modeling approach for metasurfaces with high degrees of freedom," Optics Express, vol. 28, no. 21, 2020, doi: 10.1364/oe.401960.

[29] S. An et al., "Multifunctional metasurface design with a generative adversarial network," Adv. Opt. Mater., vol. 9, no. 5, p. 2001433, 2021.

[30] H. M. El Misilmani, T. Naous, and S. K. Al Khatib, "A review on the design and optimization of antennas using machine learning algorithms and techniques," International Journal of $R F$ and Microwave ComputerÁ Aided Engineering, vol. 30, no. 10, p. e22356, 2020.

[31] A. Massa, D. Marcantonio, X. Chen, M. Li, and M. Salucci, "DNNs as applied to electromagnetics, antennas, and propagation-A review," IEEE Antennas and Wireless Propagation Letters, vol. 18, no. 11, pp. 2225-2229, 2019.

[32] P. Watson, K. Gupta, R. J. I. J. o. R. Mahajan, and M. C. A. Engineering, "Applications of knowledgeẢbased artificial n eural network modeling to microwave components," vol. 9, no. 3, pp. 254-260, 1999.

[33] P. Watson, K. Gupta, and R. Mahajan, "Development of knowledge based artificial neural network models for microwave components," in 1998 IEEE MTT-S International Microwave Symposium Digest (Cat. No. 98CH36192), 1998, vol. 1: IEEE, pp. 9-12.

[34] V. K. Devabhaktuni et al., "Neural networks for microwave modeling: Model development issues and nonlinear modeling techniques," vol. 11, no. 1, pp. 4-21, 2001.

[35] H. J. Delgado, M. H. Thursby, and F. M. J. I. t. o. n. n. Ham, "A novel neural network for the synthesis of antennas and microwave devices," vol. 16, no. 6, pp. 1590-1600, 2005.

[36] C. Zhang, J. Jin, W. Na, Q.-J. Zhang, M. J. I. T. o. M. T. Yu, and Techniques, "Multivalued neural network inverse modeling and applications to microwave filters," vol. 66, no. 8, pp. 3781-3797, 2018.

[37] J. Jin et al., "Deep neural network technique for high-dimensional microwave modeling and applications to parameter extraction of microwave filters," vol. 67, no. 10, pp. 4140-4155, 2019.

[38] W. Na, W. Zhang, S. Yan, F. Feng, W. Zhang, and Y. J. I. A. Zhang, "Automated neural network-based multiphysics parametric modeling of microwave components," vol. 7, pp. 141153-141160, 2019.
[39] F. Feng et al., "Recent advances in parametric modeling of microwave components using combined neural network and transfer function," vol. 33, no. 6, p. e2733, 2020.

[40] J. Jin et al., "Recent advances in neural network $\grave{A}$ based inverse modeling techniques for microwave applications," vol. 33, no. 6, p. e2732, 2020.

[41] J. Jin, F. Feng, J. Zhang, S. Yan, W. Na, and Q. J. I. A. Zhang, "A novel deep neural network topology for parametric modeling of passive microwave components," vol. 8, pp. 82273-82285, 2020.

[42] Z. Zhang, Q. S. Cheng, H. Chen, F. J. I. M. Jiang, and W. C. Letters, "An efficient hybrid sampling method for neural network-based microwave component modeling and optimization," vol. 30, no. 7, pp. 625-628, 2020.

[43] Q.-J. Zhang, K. C. Gupta, V. K. J. I. t. o. m. t. Devabhaktuni, and techniques, "Artificial neural networks for RF and microwave designfrom theory to practice," vol. 51, no. 4, pp. 1339-1350, 2003.

[44] Y. Cao, G. Wang, Q.-J. J. I. t. o. m. t. Zhang, and techniques, "A new training approach for parametric modeling of microwave passive components using combined neural networks and transfer functions," vol. 57, no. 11, pp. 2727-2742, 2009.

[45] G. Cybenko, "Approximation by superpositions of a sigmoidal function," Mathematics of control, signals and systems, vol. 2, no. 4, pp. 303-314, 1989.

[46] K. Hornik, M. Stinchcombe, and H. White, "Multilayer feedforward networks are universal approximators," Neural networks, vol. 2, no. 5, pp. 359-366, 1989. 